\title{
Orbital stability near the (87) Sylvia system
}

\author{
S. Aljbaae, ${ }^{1 \star}$ T. G. G. Chanut, ${ }^{1 \star}$ A. F. B. A. Prado, ${ }^{1 \star}$ V. Carruba ${ }^{\odot},{ }^{2}$ H. Hussmann, ${ }^{3}$ \\ J. Souchay ${ }^{4}$ and D. M. Sanchez ${ }^{\oplus 1}$ \\ ${ }^{1}$ Division of Space Mechanics and Control, INPE, C.P. 515, 12227-310 São José dos Campos, SP, Brazil \\ ${ }^{2}$ School of Natural Sciences and Engineering, São Paulo State University (UNESP), 12516-410 Guaratinguetá, SP, Brazil \\ ${ }^{3}$ DLR Institute of Planetary Research, Rutherfordstr. 2, D-2489 Berlin, Germany. \\ ${ }^{4}$ SYRTE, Observatoire de Paris, PSL Research University, CNRS, Sorbonne Universités, UPMC Univ. Paris 06, LNE, 61 avenue de l'Observatoire, F-75014 \\ Paris, Fr
}

Accepted 2019 April 5. Received 2019 March 15; in original form 2018 December 27

\begin{abstract}
The main goal of our work is to study the orbital dynamics of a spacecraft near the (87) Sylvia system. Here, we consider a non-homogeneous mass distribution with a dense core inside the primary asteroid. The Mascon gravity framework using the shaped polyhedral source, from light-curve data, is chosen to calculate the gravitational field. The zero-velocity curves show four unstable equilibrium points. In the absence of any solar or other celestial body perturbations, a numerical analysis of the orbital dynamics in the potential field of Sylvia is done to delineate the region of stable and unstable motions. In our model, the motions of the two moons of Sylvia and of the spacecraft are integrated with the classical equations of motion in the body-fixed frame of reference. An orbit is considered stable if the variation of its periapsis radius does not exceed a threshold value (i.e. $6 \mathrm{~km}$ ), and the variation of its eccentricity does not exceed 0.05 , although the orientation of these orbits may change. We found that the first stable orbit is detected at a distance of $550 \mathrm{~km}$ from the centre of Sylvia. No collision occurs with the central body beyond $350 \mathrm{~km}$. The collisions with Remus occur between 300 and $900 \mathrm{~km}$, while with Romulus they occur between 900 and $1450 \mathrm{~km}$. Moreover, the orbits escape from the system when the distance is smaller than $350 \mathrm{~km}$. Finally, we found that the stability region around our system decreases when the initial eccentricity increases.
\end{abstract}

Key words: gravitation-celestial mechanics-minor planets, asteroids: individual: (87) Sylvia.

\section{INTRODUCTION}

The eighth largest body in the asteroid belt, (87) Sylvia, was the first asteroid known to have more than one moon. It is a triple asteroid, discovered by N. R. Pogson on 1866 May 16 (Pogson 1866), surrounded by two satellites orbiting in nearly circular orbits in the same plane and direction, almost aligned with Sylvia's equatorial plane. The outer satellite, Romulus, was discovered in 2001 by Brown et al. (2001) using the $10 \mathrm{~m}$ Keck II telescope. This moonlet orbits Sylvia at $\sim 1356 \mathrm{~km}$ every $(3.641191 \mp$ $1.98) \times 10^{-4} \mathrm{~d}(87.388584 \mp 0.004752 \mathrm{~h})$, with an eccentricity of 0.001 . Its diameter was estimated between 14 and $22 \mathrm{~km}$ and its mass is $(9.319 \pm 5.406) \times 10^{14} \mathrm{~kg}(0.006$ percent of the mass of the primary body). The inner satellite, Remus, was

\footnotetext{
^E-mail: safwan.aljbaae@gmail.com (SA);thierry@feg.unesp.br (TGGC);
} antonio.prado@inpe.br (AFBAP) discovered in 2004 using the improved Adaptive Optics system at the European Southern Observatory's Very Large Telescope (VLT; Marchis et al. 2005). With 5-9 km in diameter, this satellite is located at $\sim 706 \mathrm{~km}$, has a period of $(1.356654 \pm 5.9) \times 10^{-5} \mathrm{~d}$ (32.559 $696 \mp 0.001416 \mathrm{~h})$, and an eccentricity of 0.016 . Its mass was estimated to be $(7.333 \pm 0.717) \times 10^{14} \mathrm{~kg}(0.005$ percent of the mass of the primary body). The triple system of Sylvia is dynamically very stable over a long time-scale (Winter et al. 2009; Vokrouhlický et al. 2010). The mass of the primary was estimated by Fang, Margot \& Rojo (2012) to be $1.484_{-0.014}^{+0.016} \times 10^{19}$ $\mathrm{kg}$, fitting the fully dynamical three-body model to the available astrometric data. The authors reported a corresponding density of $1.29 \pm 0.39 \mathrm{~g} \mathrm{~cm}^{-3}$. Combining the size with the mass estimation, Hanuš, Marchis \& Ďurech (2013) derived a higher bulk density of $1.65 \pm 0.56 \mathrm{~g} \mathrm{~cm}^{-3}$. However, Berthier et al. (2014) derived the mass of (87) Sylvia from the best-fitting Keplerian orbit of the satellites. The authors found a mass of $(1.38 \pm 0.151) \times 10^{19} \mathrm{~kg}$ with a density of $1.29 \pm 0.16 \mathrm{~g} \mathrm{~cm}^{-3}$ from the data of Remus, and a mass 
of $(1.476 \pm 0.128) \times 10^{19} \mathrm{~kg}$ with a density of $1.38 \pm 0.16 \mathrm{~g} \mathrm{~cm}^{-3}$ from Romulus data. Recently, using Adaptive Optics for (87) Sylvia and its satellite Romulus, Drummond, Reynolds \& Buckman (2016) obtained a mass of $(1.470 \pm 0.011) \times 10^{19} \mathrm{~kg}$ with a bulk density of $1.35 \pm 0.04 \mathrm{~g} \mathrm{~cm}^{-3}$. Berthier et al. (2014) and Beauvalet \& Marchis (2014) indicated the possibility of Sylvia to have a dense core embedded into an irregularly shaped material. Its low density may be related to a large macro-porosity. The primary body [(87) Sylvia] is a fairly fast rotator completing one rotation in about $5.184 \mathrm{~h}$ (Marchis et al. 2006).

(87) Sylvia is a dark body with an absolute magnitude $H=6.940$ (WISE; Masiero et al. 2011) and probably has a very primitive composition. The asteroid is a member of the Cybele group, with a perihelion of 3.298 2073 au and an aphelion distance of 3.67236316 au. The orbital period around the Sun is about $6.52 \mathrm{yr}$. (87) Sylvia is the parent body of a family in the Cybele region, which resides beyond the 2:1 Jupiter mean motion resonance. Vokrouhlický et al. (2010) considered the possibility that the Sylvia group might have been created just after the last phases of planetary migration that happened 3.8-3.9 Gyr ago, according to the Nice model (Gomes et al. 2005; Tsiganis et al. 2005; Carruba et al. 2015). This is in agreement with evolutionary time-scales of Sylvia's satellites, whose sizes are typical for other members in the family and were formed at the same time, and by the same process, as the family (Durda et al. 2004). Recently, Carruba et al. (2015) found that this family may be one of the oldest families in the extended main belt, with an age estimation of $1220 \pm 40$ Myr old, and confirmed that the orbital region of the Cybele asteroids was unstable prior to the jumping-Jupiter event. In this way, preliminary plans to launch a spacecraft up to this triple asteroid, for spectroscopy and photometric studies to determine the internal structure, will contribute to our understanding of the early history of the Solar system.

Thus, the aim of this paper is to map and characterize the orbital dynamics of a spacecraft close to (87) Sylvia, considering a model with different density layers and the existence of the two moons, which may affect the stability of some orbits around the central body as we will see later on. Our paper will have the following structure. We first present the physical properties of the polyhedral shape of the central body in Section 2. In Section 3, we discuss a possible internal structure of (87) Sylvia. Then, in Section 4, neglecting the presence of the two moons, we study the dynamical properties in the vicinity of our target calculating the Jacobi integral and we obtain the zero-velocity surfaces and the equilibrium points of the system. A general presentation of the triple system of (87) Sylvia is done in Section 5. The numerical analysis of the stability of motions in the equatorial plane of Sylvia is presented in Sections 6 and 7. Finally, our conclusions are given in Section 8.

\section{PHYSICAL PROPERTIES FROM THE POLYHEDRAL SHAPE WITH UNIFORM DENSITY}

Two shape models of the asteroid (87) Sylvia are listed in the Database of Asteroid Models from Inversion Techniques (DAMIT, ${ }^{1}$ Durech; Sidorin \& Kaasalainen 2010) as model 148 (convex with 2040 faces, Kaasalainen; Torppa \& Piironen 2002) and model 719 (non-convex with 800 faces; Marchis et al. 2006; Hanuš et al. 2013), derived from light-curve inversion method. We start this work by

\footnotetext{
${ }^{1}$ http://astro.troja.mff.cuni.cz/projects/damit
}

checking if the coordinate axis of each shape model is aligned with the principal axes of inertia, in such a way that the inertia tensor becomes a diagonal matrix. The algorithm of Mirtich (1996) is used to compute the 3D inertia tensor of the polyhedron shape with a uniform density. The inertia tensor found for both models is not diagonal. This means that the body is not oriented along its principal axes of inertia. The angle between each axis of coordinates and the direction of the principal moments of inertia are $\left(\theta_{x}=-57.95^{\circ}\right.$, $\left.\theta_{y}=57.99^{\circ}, \theta_{z}=2.23^{\circ}\right)$ for the convex model and $\left(\theta_{x}=16.20^{\circ}\right.$, $\theta_{y}=16.20^{\circ}, \theta_{z}=1.47^{\circ}$ ) for the non-convex model. As we can see, the spin axis (z-axis) is almost aligned with the maximum moment of inertia axis while the other two axes are randomly oriented. The shape of Sylvia viewed in various perspectives is presented in Fig. 1. The $x$-axis seems to be inverted between the convex and non-convex model. However, the orientation of the original model 719 is closer to the axes of its principal moments of inertia. According to Kaasalainen et al. (2002), the convex version should not be taken as a solid model of homogeneous density because the corresponding inertia tensor could be incorrect. Thus, henceforward we will use the non-convex shape model. The overall dimensions of the Sylvia non-convex shape are $(-192.608,176.102 \mathrm{~km}) \times$ $(-128.567,127.216 \mathrm{~km}) \times(-121.482,124.492 \mathrm{~km})$ in the $x-, y$ , and $z$-directions, respectively, and the polyhedral volume is $10.700616 \times 10^{6} \mathrm{~km}^{3}$ (volume-equivalent diameter of $273.403 \mathrm{~km}$ ) leading to a density of $1.373 \mathrm{~g} \mathrm{~cm}^{-3}$. This value is in a good agreement with Berthier et al. (2014).

To discuss the exterior gravitational potential, considering a uniform density, we used the algorithm of Werner (1997) to calculate the spherical harmonic coefficients $C_{\mathrm{nm}}$ and $S_{\mathrm{nm}}$ up to degree 4 (Table 1). Please notice that $C_{11}=S_{11}=0$ and $C_{21}=S_{21}=S_{22}=$ 0 . This means that the expansion of the gravitational field is fixed around the centre of mass, and the shape is precisely oriented along the principal axes of inertia (Scheeres, Williams \& Miller 2000). However, we did not use these coefficients in our analyses, but the mascon approach developed by Chanut, Aljbaae \& Carruba (2015a), based on the idea originally presented in the theses of Venditti (2013) and applied in Aljbaae et al. (2017) to calculate the exterior gravitational potential. That is more accurate than the harmonic coefficients even if these coefficients were measured up to a degree higher than 4 .

Using the algorithm of Mirtich (1996), the principal moments of inertia of Sylvia, considered as homogeneous, are calculated as

$$
\begin{aligned}
& I_{x x}=8.867 \times 10^{22} \mathrm{~kg} \mathrm{~km}^{2}, \\
& I_{y y}=1.299 \times 10^{23} \mathrm{~kg} \mathrm{~km}^{2}, \\
& I_{z z}=1.359 \times 10^{23} \mathrm{~kg} \mathrm{~km}^{2} .
\end{aligned}
$$

The moments of inertia can be used to find the equivalent ellipsoid with semimajor axes of $173.535 \times 126.988 \times 118.466 \mathrm{~km}$. The mass-distribution parameter, as introduced in Hu \& Scheeres (2004), is $\sigma=0.870$, indicating that Sylvia is close to have a prolate shape, as shown in Fig. 1.

\section{INTERNAL STRUCTURE OF SYLVIA}

(87) Sylvia was classified as a P-type by Tholen (1989) and then as an X-type asteroid by Bus \& Binzel (2002). The mineralogy composition of such objects is still debated. Different mineralogies have been suggested, including an analogy with pure nickeliron metal meteorites, aubrite meteorites, enstatite chondrites, and, possibly, organic-rich carbonaceous chondrite meteorites (Clark 

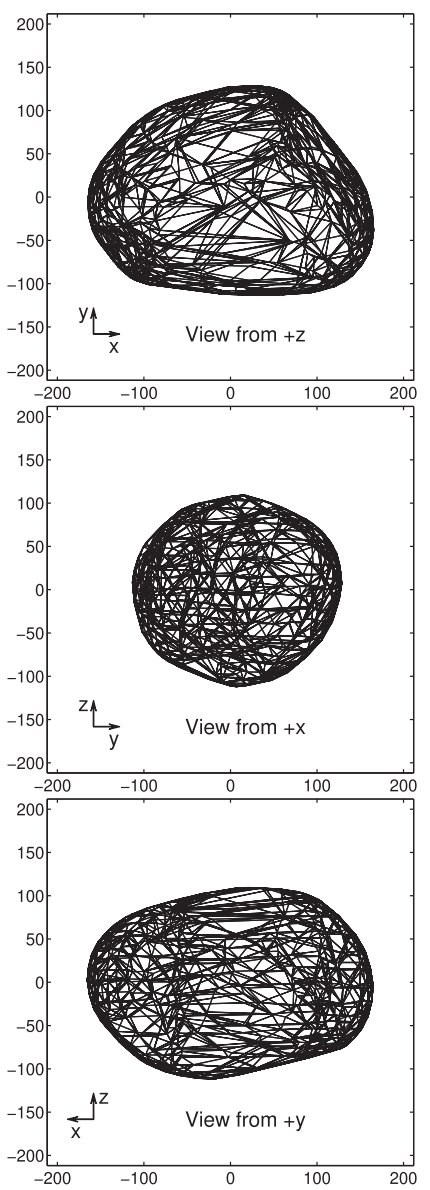
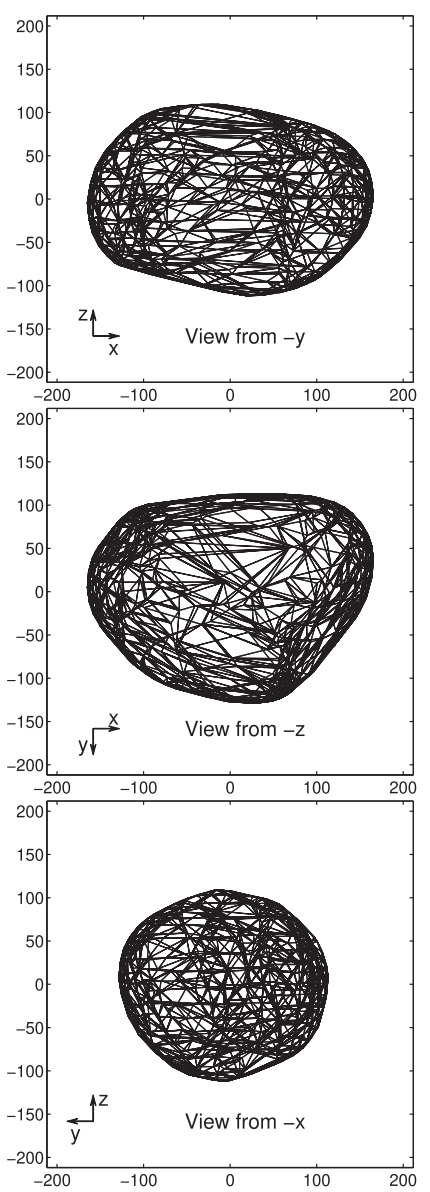
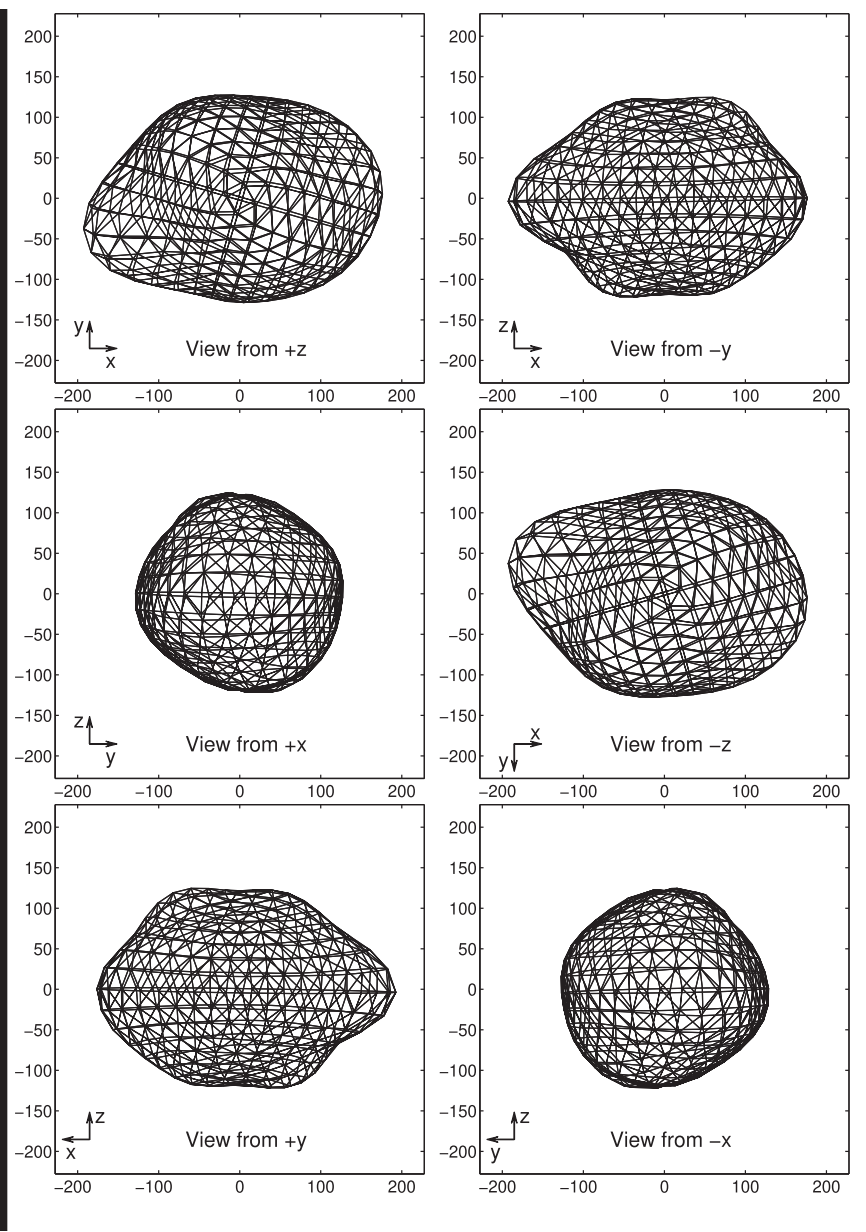

Figure 1. The polyhedral shape of (87) Sylvia (convex on the left and non-convex on the right) shown in six perspective views ( $\pm x$, $\pm y$, and $\pm z$ ), using the shape model provided by DAMIT data base with 2962 triangular faces, after aligning the asteroid with the principal axes of inertia.

Table 1. Sylvia gravity field coefficients up to order 4, using 800 triangular faces of the non-convex shape model provided by DAMIT data base. These coefficients are computed with the respect to a constant density of $1.373 \mathrm{~g} \mathrm{~cm}^{-3}$, a total mass of $1.47 \times 10^{19} \mathrm{~kg}$ (derived from the polyhedron volume), and a reference distance of $136.7015 \mathrm{~km}$.

\begin{tabular}{|c|c|c|c|}
\hline Order & Degree & $C_{\mathrm{nm}}$ & $S_{\mathrm{nm}}$ \\
\hline 0 & 0 & 1.0000000000 & - \\
\hline 1 & 0 & $2.6492480085 \times 10^{-16}$ & - \\
\hline 1 & 1 & $1.7635225962 \times 10^{-17}$ & $-1.2454772456 \times 10^{-17}$ \\
\hline 2 & 0 & $-9.7243422661 \times 10^{-2}$ & - \\
\hline 2 & 1 & $-2.4805190454 \times 10^{-16}$ & $-3.5598423081 \times 10^{-16}$ \\
\hline 2 & 2 & $3.7427290076 \times 10^{-2}$ & $-5.2987644205 \times 10^{-15}$ \\
\hline 3 & 0 & $3.0277614248 \times 10^{-3}$ & - \\
\hline 3 & 1 & $5.3539645307 \times 10^{-3}$ & $5.3675996205 \times 10^{-3}$ \\
\hline 3 & 2 & $3.7497184617 \times 10^{-4}$ & $5.6196251491 \times 10^{-4}$ \\
\hline 3 & 3 & $-4.5635516395 \times 10^{-4}$ & $-1.5576267858 \times 10^{-3}$ \\
\hline 4 & 0 & $4.3765576583 \times 10^{-2}$ & - \\
\hline 4 & 1 & $1.0328368550 \times 10^{-3}$ & $-4.9178656188 \times 10^{-3}$ \\
\hline 4 & 2 & $-3.5151360335 \times 10^{-3}$ & $-2.4531709443 \times 10^{-3}$ \\
\hline 4 & 3 & $-1.2382559193 \times 10^{-4}$ & $2.1287766573 \times 10^{-4}$ \\
\hline 4 & 4 & $2.4071820961 \times 10^{-4}$ & $2.1061368063 \times 10^{-4}$ \\
\hline
\end{tabular}

et al. 2004). Despite the lack of information on the internal structure of Sylvia, its low density could indicate high porosity in the asteroid. From 25 percent to as much as 60 percent of it may be empty space (Marchis et al. 2005). The orbits of Sylvia's two moons suggest a non-homogeneous mass distribution inside the asteroid (Berthier et al. 2014). The asteroid could be differentiated with a dense core surrounded by less dense material, as in the case of the binary system of (22) Kalliope (Vachier, Berthier \& Marchis 2012). Other differentiated asteroids such as (1) Ceres and (4) Vesta have been visited by a spacecraft $(D A W N)$. As in the case of Lutetia, studied by Aljbaae et al. (2017), we think that it is likely to expect such internal structure for asteroids as large as (87) Sylvia. To understand the effects that such a differentiation may have on the spacecraft's orbit, we refer the reader to this last paper, where the authors studied the effects of two different internal structures on the external potential of the asteroid. However, in this work, we study the dynamics in the vicinity of (87) Sylvia considering an interior structure, based on a two-layered assumption, as already used for other differentiated objects. We start from a two-layered model with a small ironstone-metal core of radius about $51 \mathrm{~km}(5.27$ percent of the total volume) and density of $3.38 \mathrm{~g} \mathrm{~cm}^{-3}$ (12.97 per cent of the total mass). This core is surrounded by a crust with a density of $1.26 \mathrm{~g} \mathrm{~cm}^{-3}$. The layers sizes and densities are constrained in 

$\begin{array}{ll}\text { Crust } & \text { Core } \\ \sim 85 \mathrm{~km}, 1.26 \mathrm{~g} . \mathrm{cm}^{-3} & \sim 51 \mathrm{~km}, 3.38 \mathrm{~g} . \mathrm{cm}^{-3}\end{array}$
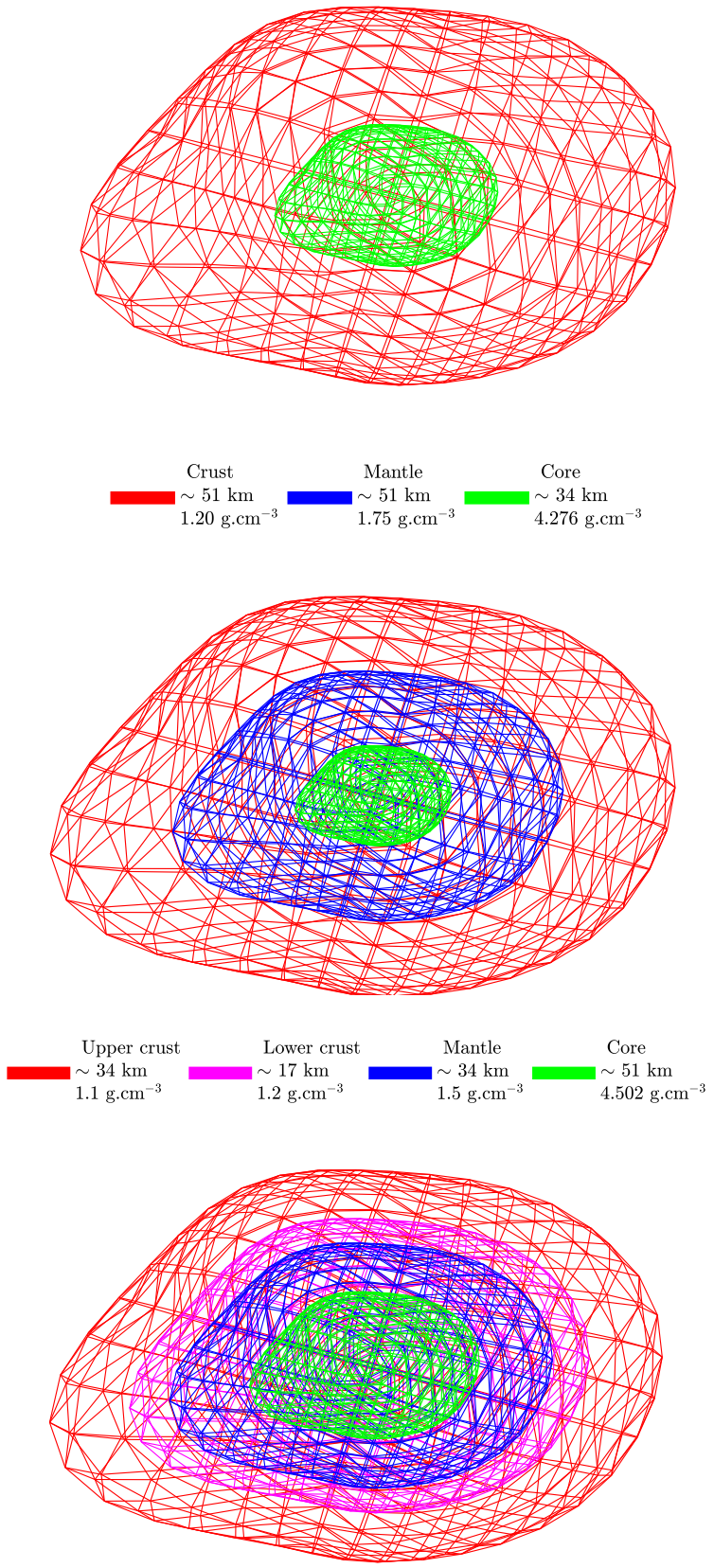

Figure 2. Assumed internal structure models of (87) Sylvia.

such way that the mean density is equal to $1.373 \mathrm{~g} \mathrm{~cm}^{-3}$. This structure (Fig. 2, top) is similar to the one discussed in Schubert et al. (2007) studying the internal structure of the Saturn's small icy moon Enceladus with a density near Sylvia's density $\left(1.608 \pm 5 \mathrm{~g} \mathrm{~cm}^{-3}\right)$. Our chosen internal structure of Sylvia seems to be in agreement with Beauvalet \& Marchis (2014) inferring the inner structure of multiple asteroid systems (45) Eugenia and (87) Sylvia from the motions of the satellites. However, other internal structures of this asteroid could be plausible. In this work, we tested two other different internal structures similar to that considered in Aljbaae et al. (2017), i.e. three- and four-layered (Fig. 2, middle and bottom). Again, the layers sizes and densities in each internal model were chosen in such a way that the mean density is equal to $1.373 \mathrm{~g} \mathrm{~cm}^{-3}$. The densities and mean thicknesses of the respective layers are shown in Fig. 2.

\section{EQUILIBRIUM POINTS}

In this section, we aim to study the orbital motion in the vicinity of (87) Sylvia, deep inside its Hill sphere, that varies between 66639.40 and $74199.12 \mathrm{~km}$ at perihelion and aphelion, respectively. In this region, the motion is highly dominated by Sylvia's own gravitational field, which allows us to safely neglect any perturbation from the remaining bodies in the Solar system, including solar perturbations. As we will see in Section 7, different internal structure models will not significantly affect the stability around our target. Thus, in this section, we first calculate the position of the equilibrium points of the gravitational potential of (87) Sylvia neglecting the presence of its two moons and considering the uniform and two-layered structure. For that purpose, we use the nonconvex shape model of Sylvia with 2962 triangular faces to calculate the gravitational potential by the Mascon approach of Chanut et al. (2015a). Considering the two-layered structure, the projection of the zero-velocity surface on to the three planes $(x y, x z$, and $y z)$ is shown in Fig. 3. The locations of the equilibrium points and their Jacobi constant $C$ are listed in Table 2. We observe that the positions of the equilibrium points $\left(E_{1}, E_{2}, E_{3}, E_{4}\right)$ move by up to 0.8730 , $1.0845,0.3442$, and $0.4593 \mathrm{~km}$, respectively, considering the twolayered structure. We then examine the stability of the equilibria calculating the eigenvalues of the linearized system (Table 3). We found that the differences in the internal structure models of the central body did not affect the stability of the equilibria, which is in agreement with Aljbaae et al. (2017). Only the exact location of the equilibrium points is affected. The saddle equilibrium points $\left(E_{1}\right.$ and $E_{2}$ ) belong to the Case 2, as defined in Jiang et al. (2014) and Wang, Jiang \& Gong (2014), whereas the centre equilibrium points ( $E_{3}$ and $E_{4}$ ) belong to the Case 5 . Thus, all the equilibrium points are unstable and the asteroid can be classified as a Type II, according to the classification originally proposed by Scheeres (1994). Then, including the two moons in our calculation, the position of the equilibrium points $\left(E_{1}, E_{2}, E_{3}, E_{4}\right)$ is moved by up to a few metres (Table 2), and the eigenvalues of the linearized system did not change. Thus, the moons did not affect significantly the stability of these points. However, they could affect some orbits near the system, as we will see in Section 6.

\section{THE TRIPLE SYSTEM OF (87) SYLVIA}

As already mentioned, this study considers the non-convex shape model of the central body provided by DAMIT data base. No shape model was found for the two moons of Sylvia. However, Berthier et al. (2014) revealed that Romulus is strongly elongated, the axis radii are $a=19 \pm 1.6 \mathrm{~km}$ and $b=7 \pm 0.4 \mathrm{~km}$. The authors estimated the quadrupole term $J_{2}$ to be equal to 0.024 . Due to lack of data, the shape of Remus is considered to be approximately spheroid with $7 \mathrm{~km}$ in diameter. In order to find an approximation of the limit of the regions where the gravity of each body (Sylvia, Remus and Romulus) is dominant, we calculate the Hill's radius for 

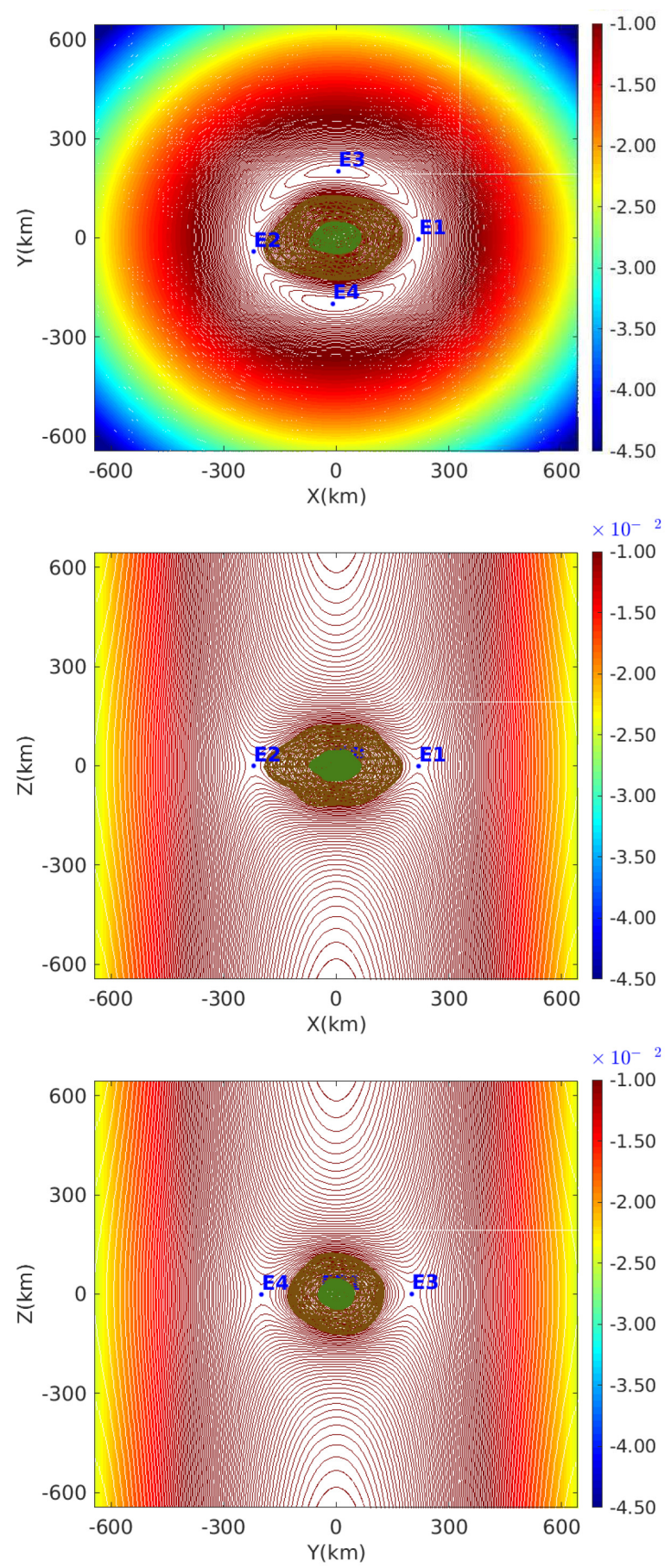

Figure 3. Zero-velocity curves and equilibrium points of (87) Sylvia in the $x y, x z$, and $y z$ planes, using the non-convex shape model. The colour code gives the intensity of the Jacobi constant in $\mathrm{km}^{2} \mathrm{~s}^{2}$. The equilibrium points outside the body $\left(E_{1}, E_{2}, E_{3}, E_{4}\right)$ are displayed in the figure.

the two-body problems composed by Sylvia-Remus and SylviaRomulus, as described in Murray \& Dermott (1999). We find the values of 18.02 , and $37.49 \mathrm{~km}$, respectively. In fact, the presence of the third body in each system could change the Hill's radius found. In Fig. 4, we represent the triple system of (87) Sylvia with the Hill's radius found for each moon. The physical characteristics and orbital elements of our system are presented in Table 4. Again, in
Table 2. Locations of the four equilibrium points of Sylvia and their Jacobi constant $C$ (using the shape model with 403 vertices).

\begin{tabular}{lrrrl}
\hline \multicolumn{1}{c}{$x(\mathrm{~km})$} & $y(\mathrm{~km})$ & $z(\mathrm{~km})$ & \multicolumn{1}{c}{$C\left(\mathrm{~km}^{2} \mathrm{~s}^{-2}\right)$} \\
\hline \multicolumn{5}{c}{ Uniform structure, without the moons } \\
$E_{1}$ & 219.967709 & -4.412460 & -1.115920 & $-0.750934 \times 10^{-2}$ \\
$E_{2}$ & -221.424048 & -41.377628 & -0.097470 & $-0.758940 \times 10^{-2}$ \\
$E_{3}$ & 5.542826 & 200.695690 & 0.232592 & $-0.705585 \times 10^{-2}$ \\
$E_{4}$ & -9.263886 & -199.301878 & -1.079508 & $-0.702781 \times 10^{-2}$ \\
\multicolumn{6}{c}{ Two-layered structure, without the moons } \\
$E_{1}$ & 219.100181 & -4.328589 & -1.063383 & $-0.748709 \times 10^{-2}$ \\
$E_{2}$ & -220.343745 & -41.282077 & -0.095917 & $-0.756271 \times 10^{-2}$ \\
$E_{3}$ & 5.450720 & 201.026230 & 0.205806 & $-0.706371 \times 10^{-2}$ \\
$E_{4}$ & -9.159521 & -199.737422 & -0.977813 & $-0.703792 \times 10^{-2}$ \\
\multicolumn{6}{c}{ Two-layered structure, with the two moons } \\
$E_{1}$ & 219.100254 & -4.328890 & -1.063387 & $-0.748719 \times 10^{-2}$ \\
$E_{2}$ & -220.343613 & -41.282135 & -0.095915 & $-0.756285 \times 10^{-2}$ \\
$E_{3}$ & 5.453331 & 201.026209 & 0.205841 & $-0.706381 \times 10^{-2}$ \\
$E_{4}$ & -9.157637 & -199.737509 & -0.977815 & $-0.703805 \times 10^{-2}$ \\
\multicolumn{5}{c}{}
\end{tabular}

this study, we expect non-homogeneous mass distribution inside the central body, as suggested by Berthier et al. (2014).

\section{EQUATIONS OF MOTION}

In the absence of any solar or other celestial body perturbations, the motion of a spacecraft orbiting our triple system is performed using a Bulirsch-Stoer variable step-size algorithm (Bulirsch \& Stoer 1966), optimized for the accuracy of $10^{-12}$, covering a period of $100 \mathrm{~d}$, that correspond to $\sim 28$ orbits of Romulus and to $\sim 74$ orbits of Remus. We consider this as sufficient to determine the final destiny of the orbits in the planning of a space mission to the system. In our model, the motion of the two moons of Sylvia (Remus and Romulus), that lie deeply within Sylvia's Hill sphere, and of the spacecraft are integrated with the classical equations of motion in the body-fixed frame of reference.

$$
\begin{aligned}
\ddot{x}_{j}-2 \omega \dot{y_{j}} & =\omega^{2} x_{j}+U_{x_{j}}+\mathcal{A}_{x}(\mathcal{P}), \\
\ddot{y_{j}}+2 \omega \dot{x_{j}} & =\omega^{2} y_{j}+U_{y_{j}}+\mathcal{A}_{y}(\mathcal{P}), \\
\ddot{z_{j}} & =U_{z_{j}}+\mathcal{A}_{z}(\mathcal{P}),
\end{aligned}
$$

where $i, j=1,2,3$ stand for the body concerned (spacecraft, Remus, and Romulus, respectively), $\left|r_{i}\right|=\sqrt{x_{i}^{2}+y_{i}^{2}+z_{i}^{2}},\left|r_{i}-r_{j}\right|$ is the distance between the bodies $i$ and $j, \omega$ is the spin rate of (87) Sylvia, and $U_{x_{j}}, U_{y_{j}}$, and $U_{z_{j}}$ are the first-order partial derivatives of the gravitation potential of the central body $U\left(x_{j}, x_{j}, x_{j}\right)$, calculated using the approach of Mascon 8 (Chanut et al. 2015a; Aljbaae et al. 2017). The vector $\mathcal{P}=\left(\mathcal{P}_{x}, \mathcal{P}_{y}, \mathcal{P}_{z}\right)$ describes the interaction between components $i$ and $j$. $\mathcal{A}$ is an instantaneous rotation that takes the vector $\mathcal{P}$ from an inertial frame into a body-fixed frame.

$$
\begin{aligned}
& \mathcal{P}_{x}=\sum_{i=1, i \neq j}^{3} \mathcal{G} m_{i}\left(\frac{x_{i}-x_{j}}{\left|r_{i}-r_{j}\right|^{3}}-\frac{x_{i}}{\left|r_{i}\right|^{3}}\right), \\
& \mathcal{P}_{y}=\sum_{i=1, i \neq j}^{3} \mathcal{G} m_{i}\left(\frac{y_{i}-y_{j}}{\left|r_{i}-r_{j}\right|^{3}}-\frac{y_{i}}{\left|r_{i}\right|^{3}}\right), \\
& \mathcal{P}_{z}=\sum_{i=1, i \neq j}^{3} \mathcal{G} m_{i}\left(\frac{z_{i}-z_{j}}{\left|r_{i}-r_{j}\right|^{3}}-\frac{z_{i}}{\left|r_{i}\right|^{3}}\right) .
\end{aligned}
$$


Table 3. Eigenvalues of the coefficient matrix of the four external equilibrium points.

\begin{tabular}{|c|c|c|c|c|}
\hline Eigenvalues & $\begin{aligned} & E_{1} \\
\times & 10^{-4}\end{aligned}$ & $\begin{array}{c}E_{2} \\
\times 10^{-4}\end{array}$ & $\begin{aligned} & E_{3} \\
\times & 10^{-4}\end{aligned}$ & $\begin{array}{c}E_{4} \\
\times 10^{-4}\end{array}$ \\
\hline \multicolumn{5}{|c|}{ Uniform structure } \\
\hline$\lambda_{1}$ & -2.5015 & -3.3355 & $+3.4129 i$ & $-1.2332+2.6251 i$ \\
\hline$\lambda_{2}$ & $+3.8889 i$ & +3.3355 & $-3.4129 i$ & $-1.2332-2.6251 i$ \\
\hline$\lambda_{3}$ & $-3.8889 i$ & $+4.1899 i$ & $-0.9499+2.5325 i$ & $+1.2332+2.6251 i$ \\
\hline$\lambda_{4}$ & $+3.7153 i$ & $-4.1899 i$ & $-0.9499-2.5325 i$ & $+1.2332-2.6251 i$ \\
\hline$\lambda_{5}$ & $-3.7153 i$ & $+4.0300 i$ & $+0.9499+2.5325 i$ & $+3.4539 i$ \\
\hline$\lambda_{6}$ & +2.5015 & $-4.0300 i$ & $+0.9499-2.5325 i$ & $-3.4539 i$ \\
\hline \multicolumn{5}{|c|}{ Two-layered structure } \\
\hline$\lambda_{1}$ & -2.4389 & -3.2695 & $+3.4093 i$ & $-1.1620+2.5973 i$ \\
\hline$\lambda_{2}$ & $+3.8654 i$ & +3.2695 & $-3.4093 i$ & $-1.1620-2.5973 i$ \\
\hline$\lambda_{3}$ & $-3.8654 i$ & $+4.1619 i$ & $-0.8733+2.5072 i$ & $+1.1620+2.5973 i$ \\
\hline$\lambda_{4}$ & $+3.6983 i$ & $-4.1619 i$ & $-0.8733-2.5072 i$ & $+1.1620-2.5973 i$ \\
\hline$\lambda_{5}$ & $-3.6983 i$ & $+4.0048 i$ & $+0.8733+2.5072 i$ & $+3.4465 i$ \\
\hline$\lambda_{6}$ & +2.4389 & $-4.0048 i$ & $+0.8733-2.5072 i$ & $-3.4465 i$ \\
\hline
\end{tabular}

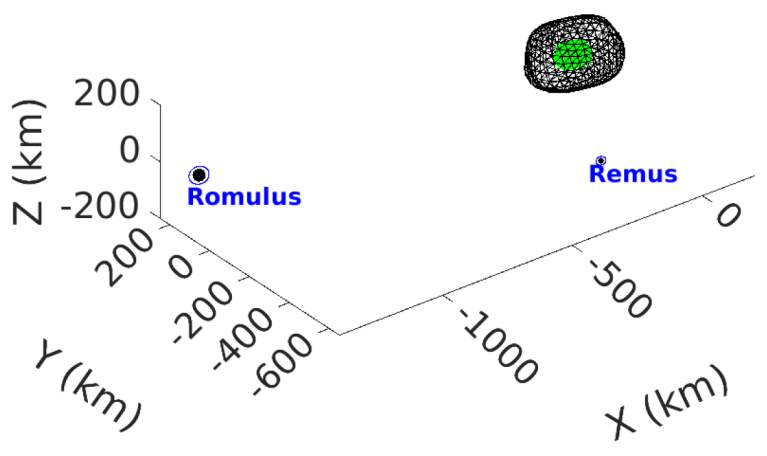

Figure 4. The triple system of asteroid (87) Sylvia and the Hill sphere radius of each moon as derived from Johnston's Asteroids with Satellites Database in 2004 September 1.

Table 4. The physical and orbital data of the triple system of Sylvia.

\begin{tabular}{lccc}
\hline & Sylvia & Remus & Romulus \\
Orbits & Sun & Sylvia & Sylvia \\
$a$ & $3.4852852 \mathrm{au}$ & $706 \mathrm{~km}$ & $1356 \mathrm{~km}$ \\
$e$ & 0.0536765 & 0.0 & 0.007 \\
$i\left({ }^{\circ}\right)$ & 9.84 & 8.6 & 8.3 \\
Period $(\mathrm{d})$ & 2381.697 & 1.356654 & 3.641191 \\
Radius $(\mathrm{km})$ & 273.403 & 7 & 18 \\
Mass $(\mathrm{kg})$ & $1.484 \times 10^{19}$ & $7.333 \times 10^{14}$ & $9.319 \times 10^{14}$ \\
Hill sphere $(\mathrm{km})$ & 70419.26 & 18.02 & 37.49 \\
\hline
\end{tabular}

For a survey of this equation, we refer the reader to Scheeres (2002, 2009) and the theses of Valéry Lainey (Lainey 2002) and Hervé Manche (Manche 2011). The $J_{2}$ of Remus and Romulus, which are estimated, respectively, to be 0.17 and 0.18 (Jiang, Baoyin \& Zhang 2017) were tested in our model. In fact, these $J_{2}$ perturbations are very small compared to the central force of our system. Consequently, ignoring them will not affect the global behaviour and stability in our simulations. The heliocentric positions of the moons without their velocities can be obtained in the equator or ecliptic plane using the Miriade ${ }^{2}$ service of the IMCCE's Virtual Observatory project (Berthier et al. 2009). However, the lack of
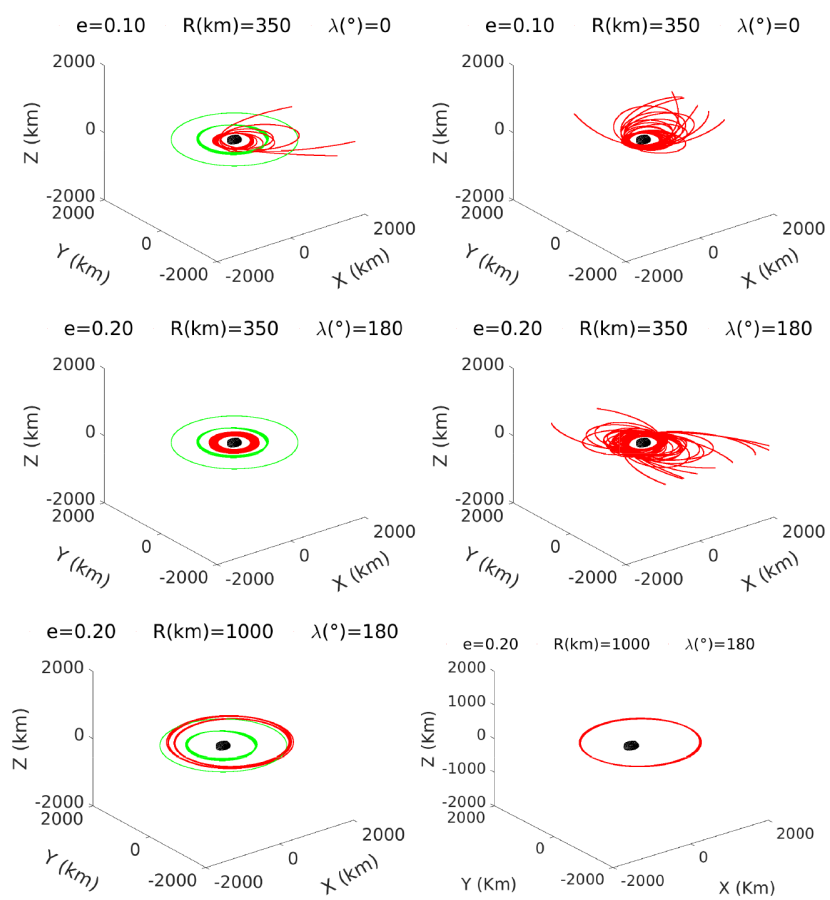

Figure 5. Some orbits affected by the two moons of Sylvia displayed on the left-hand side. The same initial conditions without the effects of the moons in the right-hand side.

information on the velocities of the moons and on the spin axis directions of the primary body make the moons' initial conditions in the body-fixed reference frame very complicated to calculate. The orbits of multiple asteroids, e.g. 87 Sylvia, are usually given for the centre of mass of the system. For those reasons, we decided to carry out initial conditions of Remus and Romulus directly from the Johnston's Asteroids with Satellites Database ${ }^{3}$ on 2004 September 1 (Fig. 4), while the initial velocities are calculated using Kepler equations. As we already mentioned in Section 4, the moons do not affect significantly the equilibrium points. However, they can

${ }^{3}$ http://www.johnstonsarchive.net/astro/astmoons/am-00087.html. Copyri ghted by Wm. Robert Johnston, 2001 

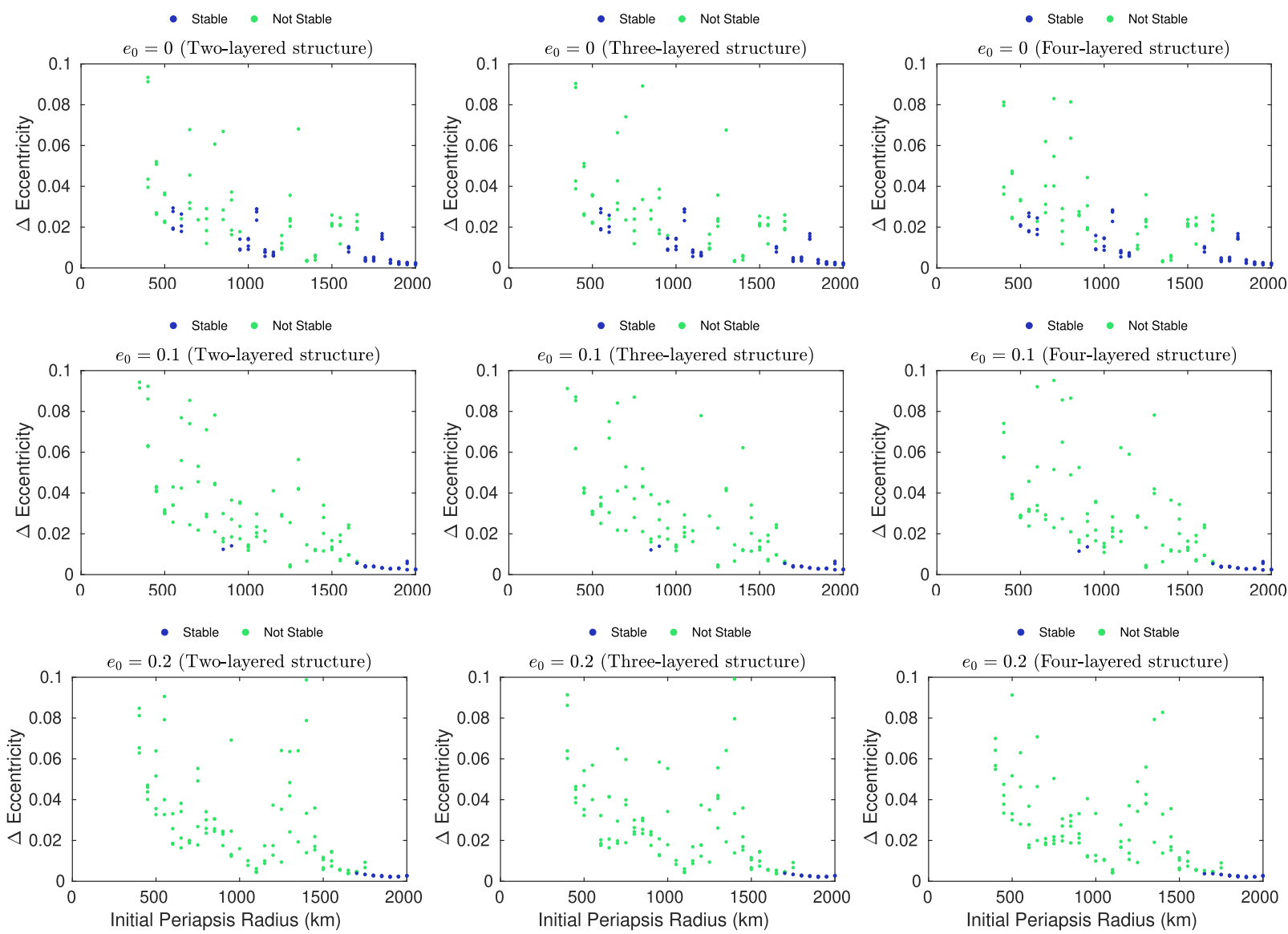

Figure 6. The variation of the eccentricities after $100 \mathrm{~d}$. Each point represents an initial condition. See the figure legend for the initial eccentricities and the internal structure applied.

Table 5. The differences in the stable orbits when different internal structures are used.

\begin{tabular}{lccc}
\hline & Two-layered & Three-layered & Four-layered \\
\hline Max. $\Delta e$ & 0.0294 & 0.0290 & 0.0284 \\
Min. $\Delta e$ & 0.0015 & 0.0015 & 0.0014 \\
\hline
\end{tabular}

significantly affect some orbits around the system. In Fig. 5, we present some orbits near the Sylvia system affected by the two moons. For this reason, we think it is necessary to include the moons in our analyses.

\section{STABILITY ANALYSIS}

Our work in this section starts by exploring the three internal models of the central body discussed in Section 3 . In the planar cases $\left(i_{\text {ini }}=\right.$ $0)$, we calculate 35 orbits with initial periapsis distance $\left(r_{\mathrm{p}}\right)$ between 250 and $2000 \mathrm{~km}$ from the centre of Sylvia. We consider three initial eccentricities $(0.0,0.1$, and 0.2$)$ with four different longitudes $\left(0^{\circ}\right.$, $90^{\circ}, 180^{\circ}, 270^{\circ}$ ). We observed the variation of the periapsis radius and the eccentricity for each orbit. We considered the orbit stable if these variations did not exceed $6 \mathrm{~km}$ and 0.05 , respectively. Our results for orbits which do not collide with the central body or the moons are presented in Fig. 6. From this figure, we conclude that the internal structure of the central body does not significantly affect the global behaviour of the stability around our triple system of Sylvia. While there are minor differences for unstable orbits in the different models, we observe that the stable ones remain the same (see Table 5 for quantifing the difference on the stable orbits). We refer the interested reader to Aljbaae et al. (2017) for detailed analyses comparing the effects of different internal structures. However, we argue that a close approach of a spacecraft to our target is necessary to fit the real gravity data to find the plausible internal structure of this asteroid.

Because of the above considerations, and since the central body could have a non-homogeneous mass distribution, in addition to the matter of computational cost, we show a detailed analysis only for the two-layered structure model of Sylvia as an example for a detailed analyses. We introduce thousands of individual massless particles distributed uniformly in the longitudes-periapsis distance plane around the triple system of asteroid Sylvia, in order to determine regions of stability. We consider 12960 particles independently orbiting the asteroid (87) Sylvia with initial periapsis distance $\left(r_{\mathrm{p}}\right)$ between 250 and $2000 \mathrm{~km}$ from the primary centre with an interval of $50 \mathrm{~km}$. In the planar cases, we study particles with initially circular $\left(e_{\text {ini }}=0\right)$ or slightly eccentric prograde orbits with initial eccentricity of $0.05,0.1$, and 0.2 .90 different longitudes $(\lambda)$ varying from $0^{\circ}$ to $356^{\circ}$ are tested. All the initial conditions are first calculated in the body-fixed reference frame from the twobody problem perturbed by the two moons. Each test particle is assumed to be at the periapsis distance in the equatorial plane of the body. For more details on the initial conditions, we refer the readers to Aljbaae et al. (2017). A discussion on the local dynamics around the triple system of Sylvia is presented in Fig. 7, where 


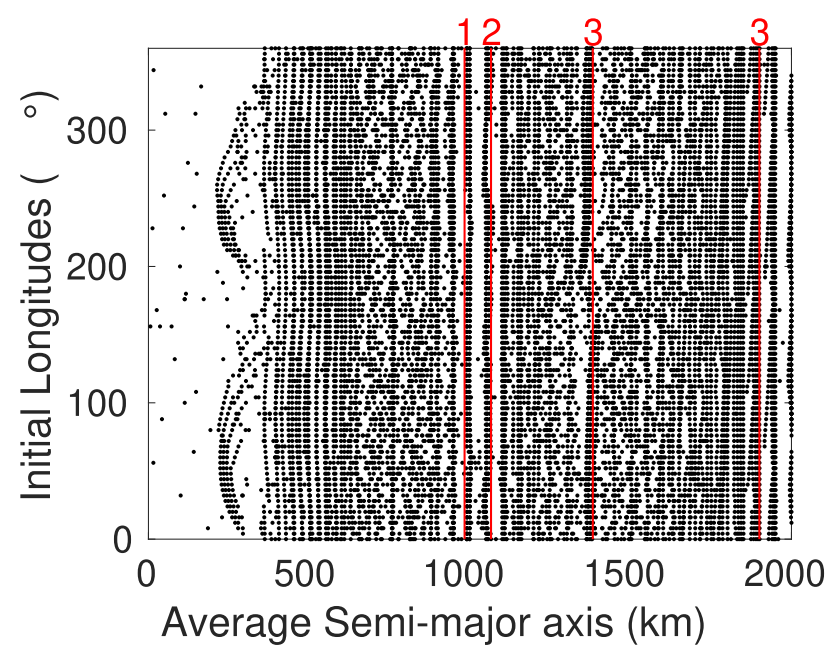

Figure 7. Average semimajor axis versus initial longitudes for massless test particles around the triple system of Sylvia.

a dynamical map in the $\left(\bar{a}, \lambda_{0}\right)$ space was computed according to the approach described in Carruba (2010). $\bar{a}$ being the average value of the semimajor axis and $\lambda_{0}$ being the initial value of the longitude. The vertical alignments of particles could be associated with certain resonances between our massless particle and the two moons (Remus and Romulus). According to Gallardo (2014), the resonance occurs when the critical angle $\left(\sigma=k_{0} \lambda_{0}+k_{1} \lambda_{1}+\right.$ $k_{2} \lambda_{2}$ ) is oscillating over time. Here, $k_{i}$ are integers and $\lambda_{i}$ are the mean longitudes of the moons: Remus and Romulus, respectively. We identified the nominal location of the 1:5:-15, 1:-8:20, and 1:3:-9 resonances at $983.25,1065.57$, and $1382.18 \mathrm{~km}$ from the centre of mass of the central body, respectively. These resonances are presented in Fig. 7 as numbers 1, 2, and 3. However, studying this phenomenon in details is beyond the scope of this work. To gain further insights on orbits in resonances, a detailed analysis of the local dynamics is needed by integrating particles in the $(a, e)$ space for a longer time that could be considered in future work.

In this analyses, we concentrate on identifying some stable orbits about (87) Sylvia. Our overall results are presented in Fig. 8. Again, an orbit is considered stable if the variation of its periapsis radius does not exceed a threshold value (i.e. $6 \mathrm{~km}$ ), and the variation of its eccentricity does not exceed 0.05 , although the orientation of these orbits may change. These values are chosen to be the double of the oscillation of Remus. We notice that large changes in these parameters can occur over a short time. The Computational Geometry Algorithms Library $\left(\mathrm{CGAL}^{4}\right)$ is used in this work to handle orbits colliding with the primary body (red points). A first approximation of a spheroid with $7 \mathrm{~km}$ and an ellipsoid with semimajor axes of $19 \mathrm{~km} \times 7 \mathrm{~km} \times 7 \mathrm{~km}$ is considered for detecting collisions with the two moons Remus and Romulus (orange and yellow points, respectively). Fig. 8 shows that the first stable orbit is detected at initial $r_{\mathrm{p}}=550 \mathrm{~km}$ for the initial eccentricity $e_{0}=$ 0 . No collisions with the central body occur beyond an initial periapsis radius of $350 \mathrm{~km}$ from the centre of Sylvia. The collisions with Remus occur between 300 and $900 \mathrm{~km}$, and the majority of collisions with Romulus occur between 900 and $1450 \mathrm{~km}$. We also present in the figure the orbits escaping from the system. The escapes occur for an initial value $r_{\mathrm{p}} \leq 350$. We can notice, from

${ }^{4}$ https://www.cgal.org/
Fig. 8, a decrease in the stability region when the initial eccentricity increases.

After eliminating orbits colliding with the central body or the moons, Fig. 9 shows the variation of the periapsis radius (left-hand side) and eccentricities (right-hand side). Our stability criterion on the periapsis radius is independent of the eccentricity, i.e. the orbit with periapsis radius variation smaller than $6 \mathrm{~km}$ is stable. However, it is not the case for the eccentricity where we can see only some stable orbits with eccentricity variation smaller than 0.05 . The figure shows that increasing the initial eccentricity decreases the variation of the eccentricity in the stable orbits due to the fact that more eccentric the orbit is, higher is its speed close to the central body. That will provide less perturbed orbits. We can also notice that, within the considered area, a large majority of orbits suffer strong perturbations. As in the case of our dynamical study about (21) Lutetia (Aljbaae et al. 2017), some objects with initial circular orbits undergo strong changes of 0.03 in eccentricity after $100 \mathrm{~d}$. These changes are not large enough to affect the stability of the objects, but could potentially be hazardous for longer time-scales. For all the initial eccentricities tested in this work, many stable orbits are found beyond an initial periapsis radius of $1600 \mathrm{~km}$ from the centre of the central body. We do not identify any stable orbit with $e_{0}=$ 0.2 close to Sylvia. However, some isolated regions of stable orbits with other initial eccentricities are found between the two moons $(850-1150 \mathrm{~km})$. Some stable low orbits found with $e_{0}=0$ occur at an initial periapsis radius of $550 \mathrm{~km}$ from the centre of Sylvia.

As an example of a stable orbit, we present in the top panel of Fig. 10 the orbit launched from the initial longitude $\lambda_{0}=88^{\circ}$, an initial eccentricity of 0.05 and initial periapsis radius of $550 \mathrm{~km}$. In the middle panel, we present an orbit launched from the initial longitude $\lambda_{0}=28^{\circ}$, initial eccentricity of 0.05 , and initial periapsis radius of $1650 \mathrm{~km}$. In these two cases, the periapsis radius and eccentricity are constant on average. For the first case, a resonance phenomenon occurs. Again, a detailed analysis of this type of orbits should be investigated in future studies. The bottom panel presents an example of unstable orbit launched from the initial longitude $\lambda_{0}=160^{\circ}$, the initial eccentricity of 0.0 , and initial periapsis radius of $700 \mathrm{~km}$. The periapsis radius and eccentricity in such motion are rapidly departing from their initial values. The unstable orbits will usually end by impact or escape from the system.

For further analysis of the orbits shown in Fig. 10, we present, in the left-hand and middle panels of Fig. 11, the orbital mechanical energy relative to the rotating asteroid $(H)$ and relative to the inertia space $(M)$, respectively, while the right-hand panels give the distance between the particle/spacecraft to Remus (the inner satellite of the system of Sylvia). We used the standard definition of the full twobody energy perturbed by the shape of the central body and the existence of the two moons of Sylvia as presented in Scheeres et al. (1996, 2000) and Chanut et al. (2015b). Thus, from our equation of motion given in Section 6, the mechanical energy of the orbits can be calculated by the next formula

$$
\begin{aligned}
& H=\frac{1}{2}\left(\dot{x}^{2}+\dot{y}^{2}+\dot{z}^{2}\right)-\frac{1}{2} \omega^{2}\left(x^{2}+y^{2}\right)-U, \\
& M=\frac{1}{2} v^{2}-U,
\end{aligned}
$$

where $x, y, x$, and $\dot{x}, \dot{y}, \dot{x}$ are the location and velocity of the particle in the body-fixed frame of reference. $v$ is the relative velocity of the particle relative to the inertia space. We recall here that the transformation between the body-fixed and the inertial frame is made by a simple rotation around $z$-axis. $U$ is the gravitational 

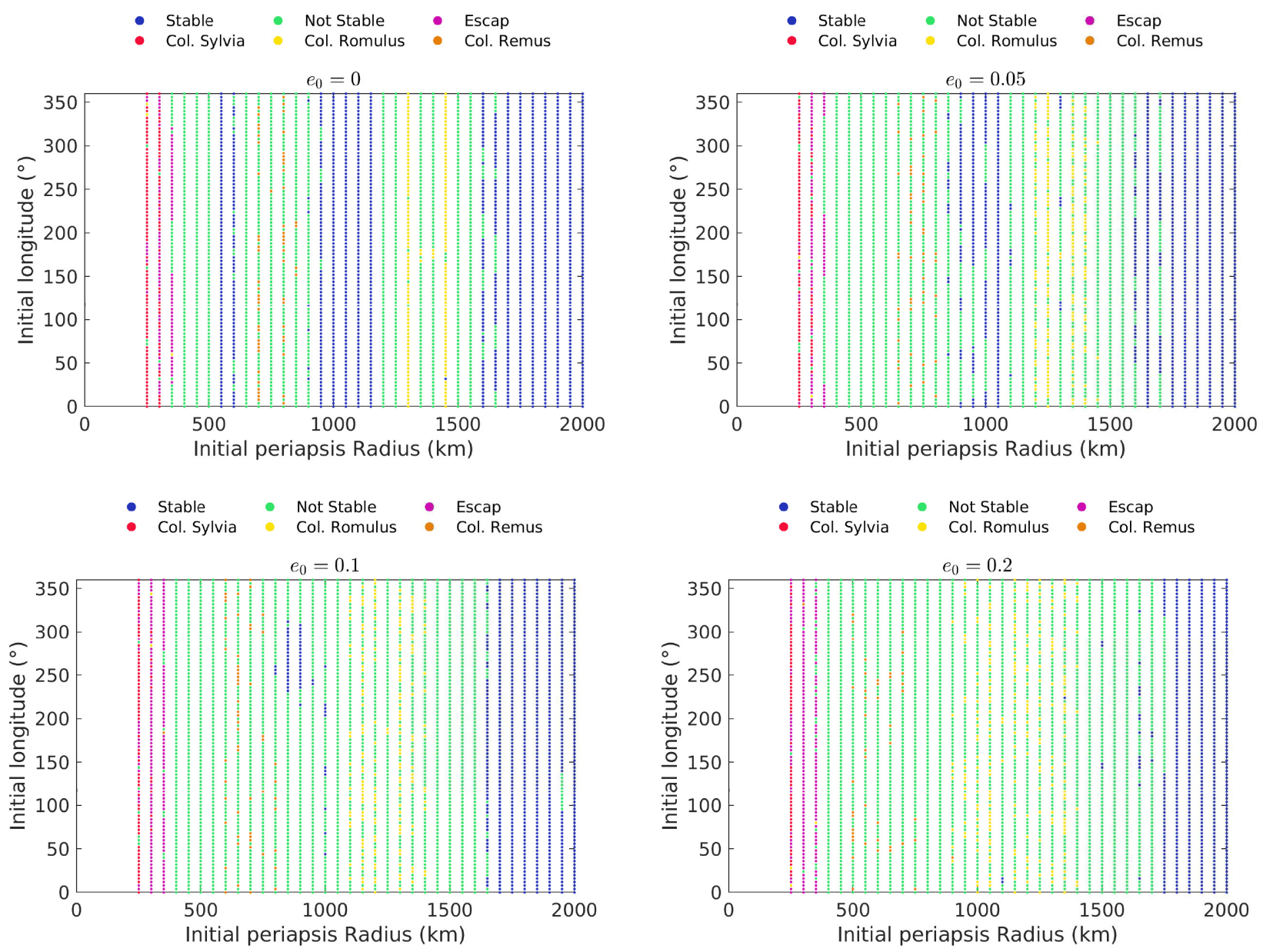

Figure 8. Stability maps of equatorial orbits relative to (87) Sylvia with initial eccentricities $e_{0}=0,0.05,0.1$, and 0.2 after $100 \mathrm{~d}$. The initial longitudes go from $0^{\circ}$ up to $360^{\circ}$. The initial periapsis radius are from 250 to $2000 \mathrm{~km}$.

potential of the asteroid calculated by the Mascon approach of Chanut et al. (2015a) considering the existence of the two moons of Sylvia as demonstrated in Scheeres $(2002,2009)$ and we applied in our equation of motion. From Fig. 11, we can notice that the energies for the first two orbits are constant on average and change periodically. As expected, the variations of the energies match those of $a$ and $e$. That is due to the close approach with the inner moon of the system, as shown in the right-hand panels of Fig 11. In fact, the third orbit of Fig. 10 is the closest one to Remus, so its mean motion is very close to that of Remus. The orbit is unstable, but it did not intersect with Remus. Thus, this type of orbit could be suitable to observe the inner moonlet during the close approach. It is worth to mention here that Remus may act as a shepherd moon, cleaning its vicinity from natural particles. However, this point needs to be more investigated in future studies.

\section{CONCLUSION}

The main goal of this work was to determine the orbital dynamics of a spacecraft around the triple asteroidal system gathering (87) Sylvia and its two moons Remus and Romulus. Considering a non-homogeneous mass distribution with a dense core inside the primary asteroid, we used the Mascon gravity approach, developed by Chanut et al. (2015a), to provide a detailed numerical analysis describing the orbital dynamics associated with this triple asteroidal system. Our approach (mascon) uses the polyhedral shape of the asteroid, dividing the body into eight equal layers, to calculate the exterior gravitational potential, which is more accurate than the spherical harmonic coefficient model, even if these coefficients were measured up to a very high degree and order. Our study started by obtaining the physical properties of the polyhedral-shaped asteroid (87) Sylvia and constraining the possible internal structure of this asteroid, suggesting three interior structure models, based on other observed differentiated small bodies. Then, we studied the dynamical properties in the vicinity of Sylvia by calculating the Jacobi integral, the zero-velocity surfaces and the equilibrium points of the system. Examining the stability of the equilibria, we found that all the equilibrium points are unstable and the asteroid can be classified as a Type II according to Scheeres (1994). In the absence of any solar or other celestial body perturbations, the motion of a spacecraft orbiting our system is modelized using the Bulirsch-Stoer variable step-size algorithm, over a period of $100 \mathrm{~d}$. That is enough to determine the final destiny of the orbits for a possible space mission. We tested the effects of three different internal structure models of the central body on orbits around the system and we found that these structures did not affect the global behaviour of the stability. In this aspect, we presented a complete analysis considering the two-layered structure.

In our model, the motions of the two moons and of the spacecraft were integrated with the classical equations of motion with respect 


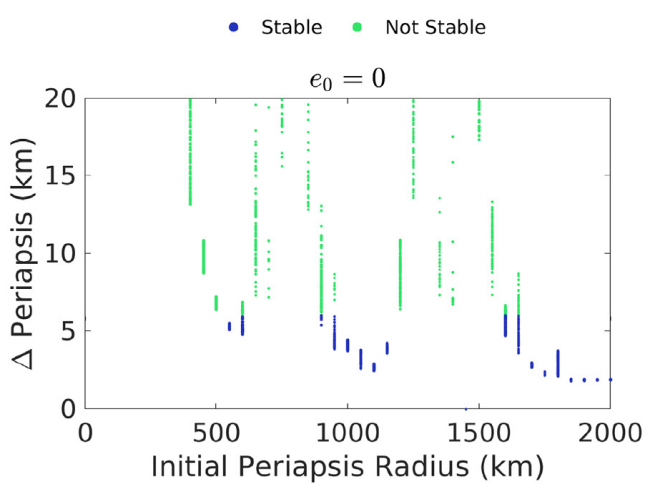

- Stable - Not Stable

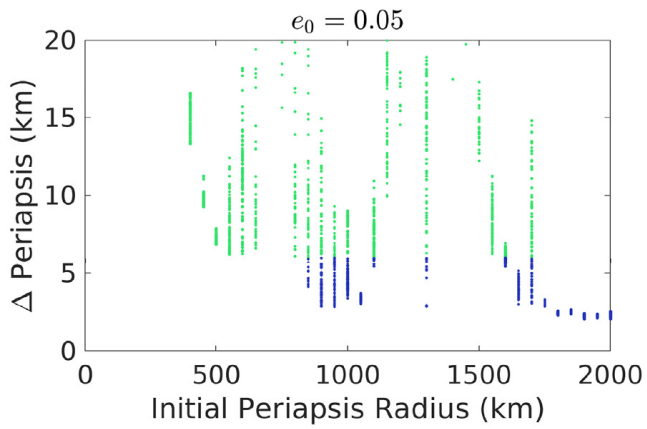

- Stable - Not Stable

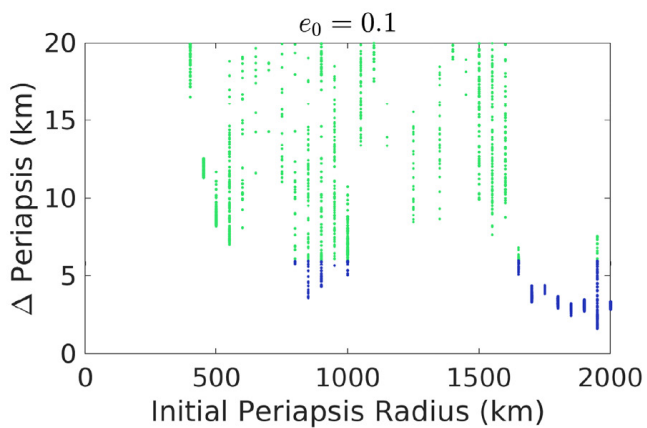

- Stable - Not Stable

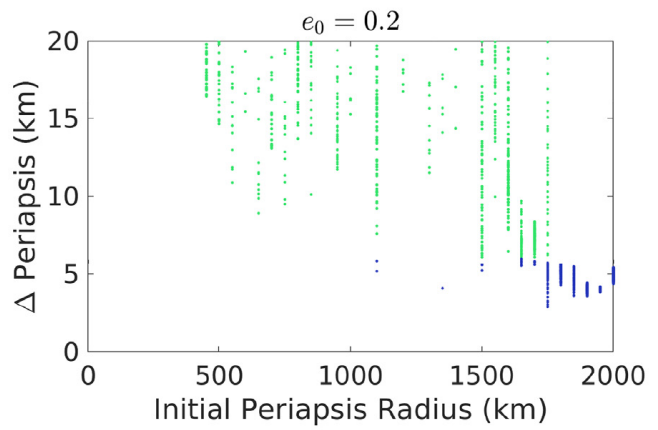

- Stable Not Stable

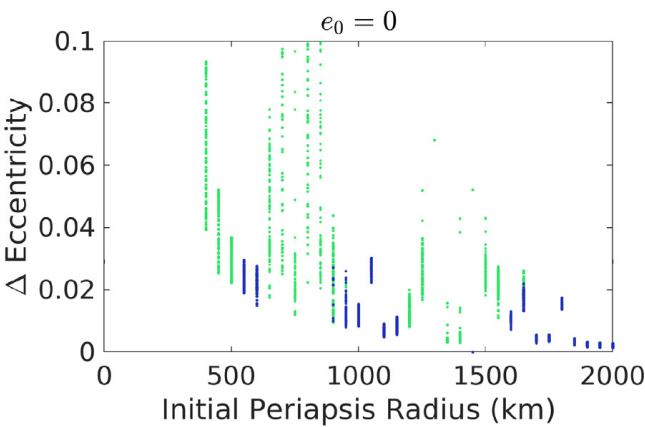

- Stable - Not Stable

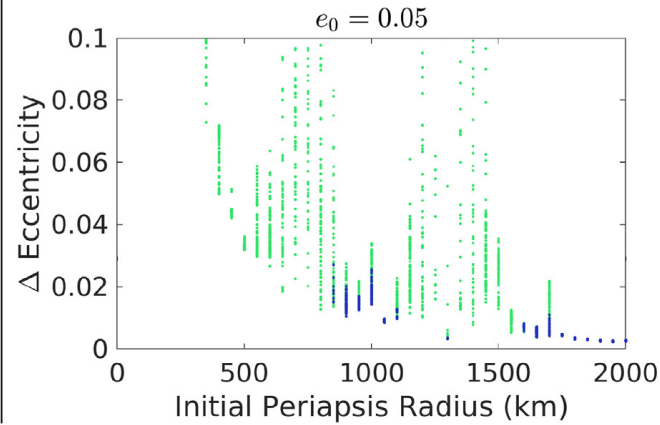

- Stable - Not Stable

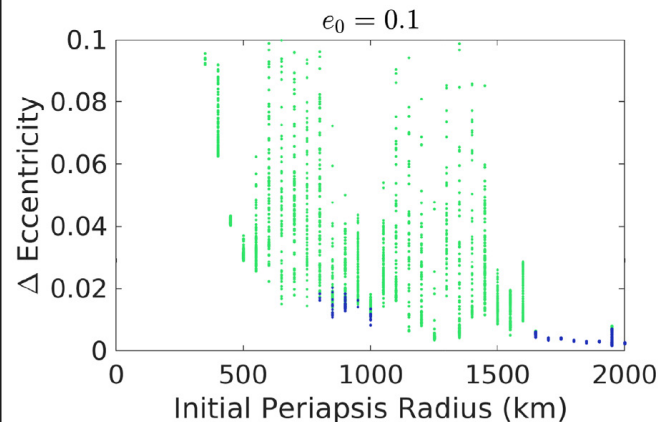

- Stable - Not Stable

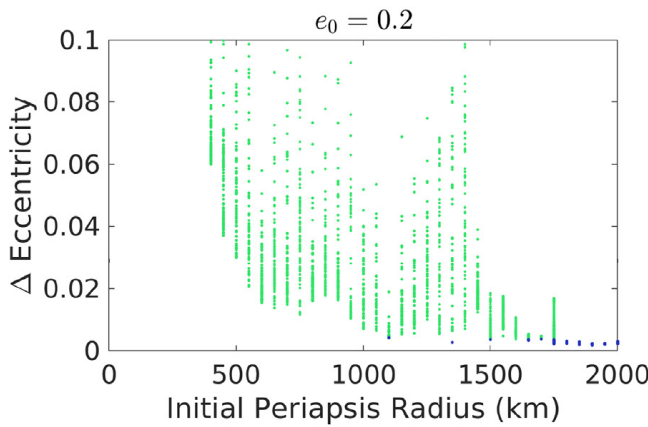

Figure 9. The variation of periapsis radius (left-hand side) and eccentricities (right-hand side). Each point represents an initial condition.

to a body-fixed frame of reference. 12960 prograde orbits with periapsis distance between 250 and $2000 \mathrm{~km}$ from the centre of the central body with an interval of $50 \mathrm{~km}$ were integrated considering initially circular $\left(e_{\mathrm{ini}}=0\right)$ or slightly eccentric orbits with initial eccentricities of $0.05,0.1$, and 0.2 . The initial conditions of each orbit were chosen in such a way that the spacecraft was initially located at the periapsis distance on the equatorial plane with 90 different longitudes. A dynamical map in the (average semimajor 

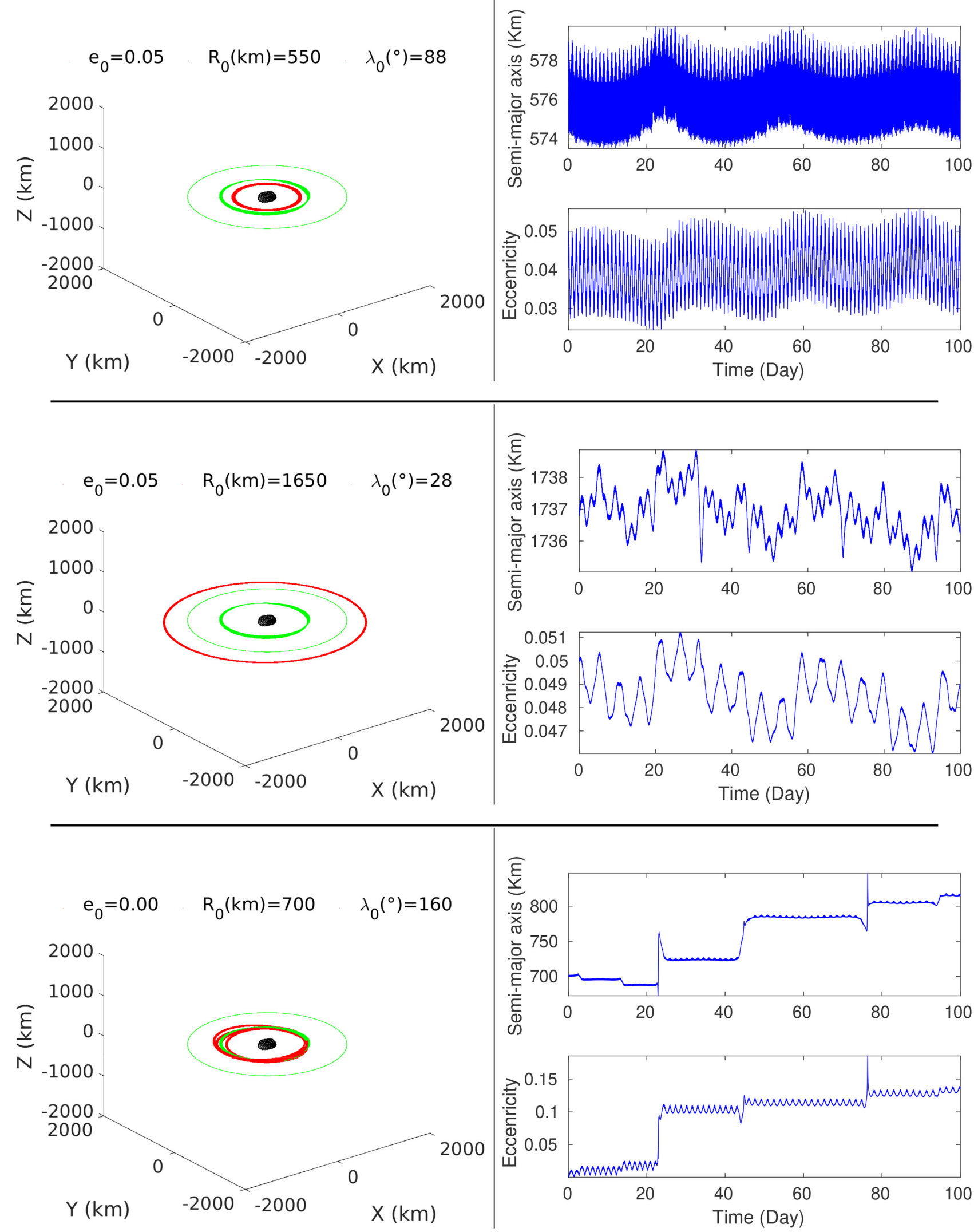

Figure 10. Example of equatorial orbits in the Sylvia-fixed frame over $100 \mathrm{~d}$. 


$$
\mathrm{e}_{0}=0.05 \quad \mathrm{R}_{0}(\mathrm{~km})=550 \quad \lambda_{0}\left({ }^{\circ}\right)=88
$$
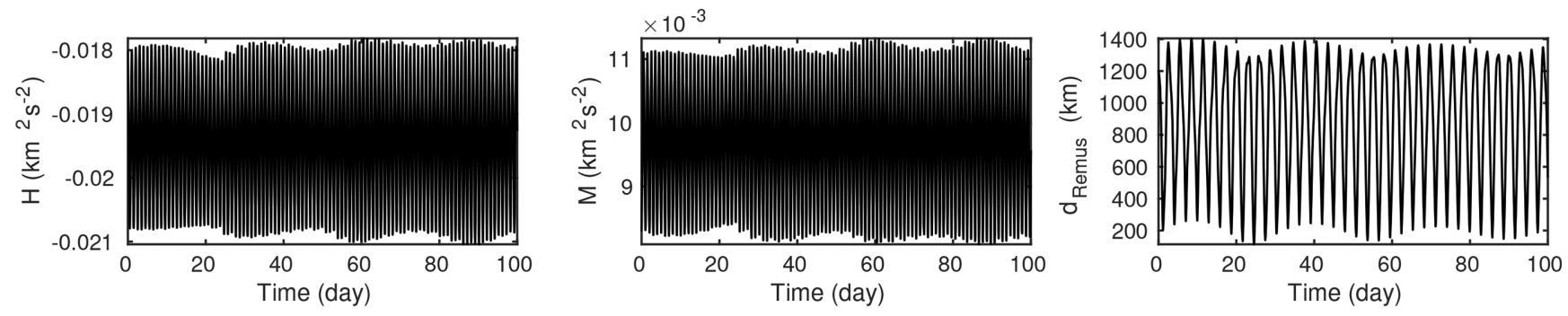

$e_{0}=0.05$

$R_{0}(k m)=1650$

$\lambda_{0}\left({ }^{\circ}\right)=28$
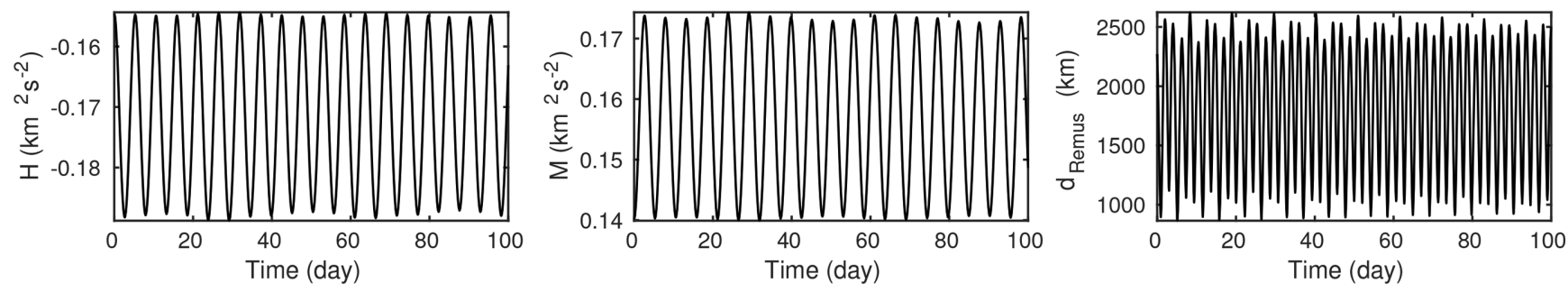

$\mathrm{e}_{0}=0.00$

$\mathrm{R}_{0}(\mathrm{~km})=700$

$\lambda_{0}\left({ }^{\circ}\right)=160$
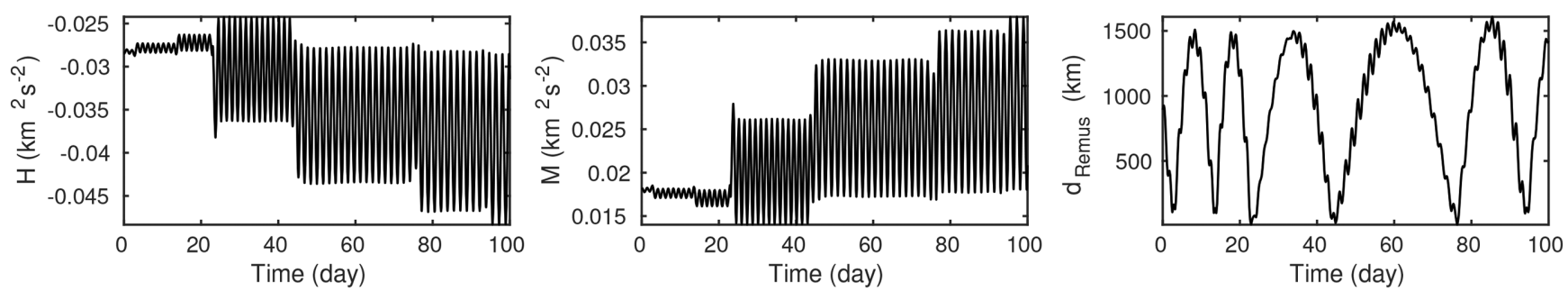

Figure 11. The behaviour of the mechanical energy relative to the asteroid (right) and relative to the inertia space (left) of the orbits in Fig. 10.

axis, initial longitudes) space was constructed, noting the presence of a resonance phenomenon, which appears as aligned particles. Further insights on this aspect should be addressed in future work. Based on observing the oscillation of the periapsis and eccentricity of each orbit, a numerical criterion was proposed in this work to identify stable orbits. The stability region increases inversely with the initial eccentricity. The first stable orbit detected at a periapsis of $550 \mathrm{~km}$. No collisions with the central body occur beyond $350 \mathrm{~km}$. The collisions with Remus occur between 300 and $900 \mathrm{~km}$, and the majority of collisions with Romulus occur between 900 and $1450 \mathrm{~km}$. We do not investigate the stability of retrograde or polar orbits in this work, as these are well known to be more stable. However, future applications of our study would be very interesting. Finally, It is worth to mention that, in our opinion, a close approach of a spacecraft with the system of Sylvia could be necessary to fit the real gravity data in order to validate or not our assumption of the internal structure of the central body.

\section{ACKNOWLEDGEMENTS}

The authors would like to thank Dr. Jerome Berthier from IMCCE at Paris Observatory for the fruitful discussions on the initial conditions of the moons of Sylvia. We would also like to thank Dr. Corinne Blondel from the Université Paris Diderot (Paris 7) for providing some useful tools for this work. The authors wish to thank the National Council for Scientific and Technological Development (CNPq), which supported this work via the grants 153683/20180, 301577/2017-0, 406841/2016-0, and 301338/2016-7. We are grateful to the financial support from the São Paulo State Science Foundation (FAPESP) via the grants 2014/22295-5, 2016/24561-0, and 2016/14665-2, and to the Coordination for the Improvement of Higher Education Personnel (CAPES).

\section{REFERENCES}

Aljbaae S., Chanut T. G. G., Carruba V., Souchay J., Prado A. F. B. A., Amarante A., 2017, MNRAS, 464, 3552

Beauvalet L., Marchis F., 2014, Icarus, 241, 13

Berthier J., Hestroffer D., Carry B., Vachier F., Lainey V., Emelyanov N. V., Thuillot W., Arlot J. E., 2009, in epsc. conf..p. 676

Berthier J., Vachier F., Marchis F., Durech J., Carry B., 2014, Icarus, 239, 118

Brown M. E., Margot J. L., Keck W. M., II, de Pater I., Roe H., 2001, IAUC, 7588,1 
Bulirsch R., Stoer J., 1966, Numer. Math., 8, 1

Bus S. J., Binzel R. P., 2002, Icarus, 158, 146

Carruba V., 2010, MNRAS, 408, 580

Carruba V., Nesvorný D., Aljbaae S., Huaman M. E., 2015, MNRAS, 451, 244

Chanut T. G. G., Aljbaae S., Carruba V., 2015a, MNRAS, 450, 3742

Chanut T. G. G., Winter O. C., Amarante A., Araújo N. C. S., 2015b, MNRAS, 452, 1316

Clark B. E., Bus S. J., Rivkin A. S., Shepard M. K., Shah S., 2004, AJ, 128, 3070

Drummond J. D., Reynolds O. R., Buckman M. D., 2016, Icarus, 276, 107

Durda D. D., Bottke W. F., Enke B. L., Merline W. J., Asphaug E., Richardson D. C., Leinhardt Z. M., 2004, Icarus, 167, 382

Durech J., Sidorin V., Kaasalainen M., 2010, A\&A, 513, A46

Fang J., Margot J.-L., Rojo P., 2012, AJ, 144, 70

Gallardo T., 2014, Icarus, 231, 273

Gomes R., Levison H. F., Tsiganis K., Morbidelli A., 2005, Nature, 435, 466

Hanuš J., Marchis F., Ďurech J., 2013, Icarus, 226, 1045

Hu W., Scheeres D. J., 2004, Planet. Space Sci., 52, 685

Jiang Y., Baoyin H., Li J., Li H., 2014, Ap\&SS, 349, 83

Jiang Y., Baoyin H., Zhang Y., 2017, Earth Moon Planets, 119, 65

Kaasalainen M., Torppa J., Piironen J., 2002, Icarus, 159, 369

Lainey V., 2002, PhD thesis, Paris Observatory

Manche H., 2011, PhD thesis, Paris Observatory

Marchis F., Descamps P., Hestroffer D., Berthier J., 2005, Nature, 436, 822

Marchis F., Kaasalainen M., Hom E. F. Y., Berthier J., Enriquez J., Hestroffer D., Le Mignant D., de Pater I., 2006, Icarus, 185, 39
Masiero J. R. et al., 2011, ApJ, 741, 68

Mirtich B., 1996, J. Graph. Tools, 1, 2

Murray C. D., Dermott S. F., 1999, Solar System Dynamics, Cambridge Univ. Press, Cambridge

Pogson N., 1866, MNRAS, 26, 311

Scheeres D. J., 1994, Icarus, 110, 225

Scheeres D. J., 2002, Celest. Mech. Dyn. Astron., 83,, 155

Scheeres D. J., 2009, Celest. Mech. Dyn. Astron., 104, 103

Scheeres D. J., Ostro S. J., Hudson R. S., Werner R. A., 1996, Icarus, 121, 67

Scheeres D. J., Williams B. G., Miller J. K., 2000, J. Guid. Control Dyn., 23,466

Schubert G., Anderson J. D., Travis B. J., Palguta J., 2007, Icarus, 188, 345

Tholen D. , 1989, in Binzel R. P., Gehrels T., Matthews M. S., eds, Asteroids II. Univ. Arizona Press, Tucson, 1139

Tsiganis K., Gomes R., Morbidelli A., Levison H. F., 2005, Nature, 435, 459

Vachier F., Berthier J., Marchis F., 2012, A\&A, 543, A68

Venditti F. C. F., 2013, PhD thesis, INPE, Sao José dos Campos

Vokrouhlický D., Nesvorný D., Bottke W. F., Morbidelli A., 2010, AJ, 139, 2148

Wang X., Jiang Y., Gong S., 2014, Ap\&SS, 353, 105

Werner R. A., 1997, Comput. Geosci., 23, 1071

Winter O. C., Boldrin L. A. G., Vieira Neto E., Vieira Martins R., Giuliatti Winter S. M., Gomes R. S., Marchis F., Descamps P., 2009, MNRAS, 395,218

This paper has been typeset from a $\mathrm{T}_{\mathrm{E}} \mathrm{X} / \mathrm{LT} \mathrm{E} \mathrm{X}$ file prepared by the author. 